\title{
La relación entre actitud y el éxito del feedback correctivo escrito en la adquisición de una segunda lengua
}

The relationship between attitude and success of written correction feedback in the acquisition of a second language

A relação entre atitude e o sucesso do feedback corretivo escrito na aquisição de uma segunda língua

Jorge LILLO

(Universidade Católica da Santíssima Concepción, Chile)

\section{RESUMEN}

El presente artículo informa parte de una tesis doctoral que investigó la actitud que tienen los estudiantes en relación al feedback correctivo (FC) escrito en una segunda lengua (L2). La actitud fue medida con un cuestionario que utilizó una escala Likert de 5 puntos. Las preferencias se midieron en porcentajes de frecuencia de respuesta y la correlación entre la actitud y el efecto del FC escrito se realizó utilizando el índice de correlación de Pearson. Además, se realizó un análisis de covarianza de medidas repetidas con la actitud como covariable para mayor precisión estadística. Los resultados indican que los estudiantes prefieren ser corregidos en la producción de textos escritos en inglés como L2 y que la actitud puede llegar a tener un efecto mediador sobre el efecto del FC.

Palabras-clave: feedback correctivo; adquisición de segundas lenguas; diferencias individuales. 


\section{ABSTRACT}

This paper reports part of a doctoral dissertation that investigated learner attitudes towards written corrective feedback (CF) in a second language (L2). Attitude was measured with a questionnaire that used a 5-point Likert scale. Preferences were measured in percentages per question and the correlation between attitude and the effect of CF using Pearson correlation index. In adition, to determine more accurately the statistical relationship between attitude and the effect of $C F$, a repeated measures analysis of covariance with attitude as covariate was performed. The results indicate that students prefer to be corrected in the production of texts in English as a second language and that attitude can mediate the effect of $C F$.

Key-words: corrective feedback; second language acquisition; individual differences.

\section{RESUMO}

Este artigo é parte duma tese de doutorado que investigou a atitude que os alunos têm em relação ao feedback corretivo (FC) escrito numa segunda língua (L2). A atitude foi medida com um questionário que incluía uma escala de Likert de 5 pontos. As preferências foram medidas em resposta à frequência de percentagens e a correlação entre a atitude e o efeito do FC escrito foram realizados utilizando o índice de correlação de Pearson. Além disso, foi realizada uma análise de covariância para medidas repetidas com atitude como uma co-variável para maior precisão estatística. Os resultados indicam que os alunos preferem ser corrigidos na produção de textos escritos em Inglês como L2 e atitude pode ter um efeito mediador sobre o efeito da FC.

Palavras-chave: feedback correctivo; aquisição de segunda língua; diferenças individuais.

\section{Introducción}

La investigación en Feedback Correctivo (FC) ha producido resultados mixtos en relación a la efectividad de la corrección de los errores gramaticales. Una de las razones puede ser que las diferencias individuales (DI) tales como la aptitud lingüística, la ansiedad y la actitud 
influyen en la recepción de la corrección, así como en la efectividad del feedback (Sheen, 2011). Por consiguiente, uno de los aspectos a considerar para entender el rol que tiene el feedback correctivo en el aprendizaje y la adquisición de una L2 es determinar si las DI influyen en los efectos de distintos tipos de FC. Uno de los propósitos del estudio ha consistido en explorar precisamente el impacto que tiene la actitud en el efecto del FC medida por la precisión gramatical de dos estructuras; a saber, pasado simple (PT) y concordancia sujeto verbo (SVA).

\subsection{El Feedback Correctivo en Adquisición de Segundas}

\section{Lenguas}

\subsubsection{El concepto de error en ASL}

Para poder comprender el FC y en especial el escrito y cuáles son los propósitos de entregar feedback acerca de los errores lingüísticos de los estudiantes, es de imperiosa necesidad comprender cómo se origina el concepto de error en la adquisición de una segunda lengua y como ha sido interpretado en años posteriores.

Los estudiantes de segundas lenguas cometen errores de comprensión y producción, los que son considerados como formas no deseadas de la lengua (Ellis, 1994). Estas formas han sido analizadas en el tiempo con diversas metodologías. Una de ellas ha sido el Análisis Contrastivo (AC), que tomaba como base de este fenómeno la interferencia que se produce desde la lengua materna al adquirir una L2. Esta metodología se concentró en explicar el fenómeno estudiando ambas lenguas en forma independiente de la producción de los hablantes, como se ilustra en la figura 1.

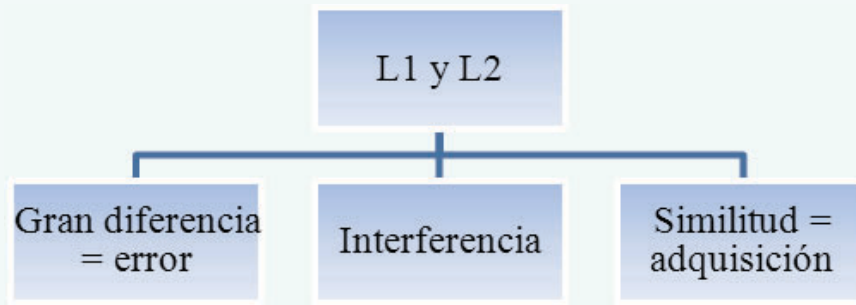

Figura 1 - Modelo de análisis contrastivo 
De esta forma se trataba de predecir los errores estudiando las diferencias y similitudes lingüísticas entre la L1 y la L2. Sin embargo y debido a no considerar lo que los estudiantes son capaces de producir en una segunda lengua, el AC dio paso a una nueva metodología en la temática del error, a saber, el Análisis de Errores (AE), que se caracteriza por estudiar la lengua de los hablantes, su interlengua ${ }^{1}$, de esta manera conocer más acerca del proceso de adquisición, mediante el estudio de los errores, con el objeto de mejorar los métodos de aprendizaje (ver fig. 2).

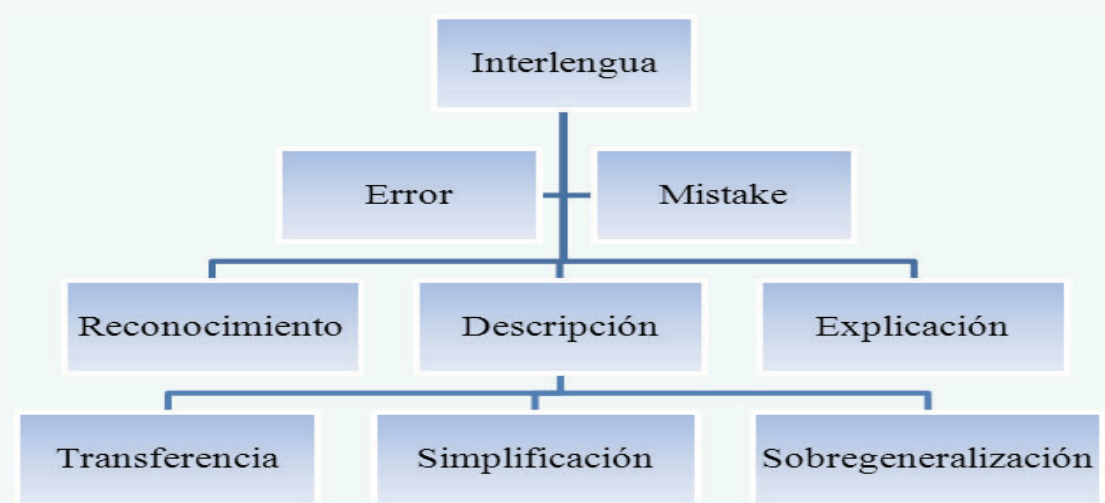

Figura 2 - Modelo de análisis de errores

Corder (1967) define el error como una desviación sistemática y consistente que caracteriza el sistema lingüístico de un hablante en un nivel determinado, lo considera un hecho inevitable y positivo: demuestra que el estudiante aprende y caracteriza su interlengua. Los conceptos más importantes introducidos por Corder son los siguientes:

1. Es el alumno quien determina el input. El profesor puede presentar una forma lingüística, pero esto no es necesariamente

\footnotetext{
1. Una interlengua es un sistema lingüístico emergente que ha sido desarrollado por el que aprende una segunda lengua, quien no ha llegado a ser completamente proficiente en la lengua meta, pero está en vías de serlo. El concepto de interlengua se fundamenta en la teoría de que existe una estructura psicológica en el cerebro humano la que se activa cuando se intenta aprender una segunda lengua (Selinker, 1972).
} 
el input, sino simplemente lo que está disponible para ser aprendido.

2. Corder introdujo la distinción entre errores sistemáticos y no sistemáticos. Los errores no sistemáticos ocurren en nuestra lengua materna; Corder llama a estos errores "mistakes" y establece que no son significativos para el proceso de aprendizaje de la lengua. Mantiene el término "errors" para los que son sistemáticos y que ocurren en una segunda lengua.

3. Cuando el estudiante ha cometido un error, la manera más eficiente para enseñarle la forma correcta es permitiéndole descubrirla a medida que prueba diferentes hipótesis.

4. Muchos errores son el resultado de la lengua materna. Corder estima que la lengua materna es facilitadora y que por lo tanto los errores son evidencia de las estrategias de aprendizaje.

Corder se basó en el trabajo de Carroll (1961) para mostrar que la forma más eficaz de enseñar a un estudiante la forma lingüística correcta es dejarlo que ponga a prueba varias hipótesis $y$, que eventualmente encuentre la forma correcta. De acuerdo a lo anterior, la autocorrección sería importante y tal vez deseada por sobre la corrección del profesor, sin embargo esto no significa que ésta deba ser eliminada porque es el profesor quien tiene la labor de enseñar las reglas de la L2 cautelando que no se corrija absolutamente cada uno de los errores a medida que estos vayan ocurriendo, porque esta acción podría traer consecuencias negativas en el proceso de aprendizaje de la L2.

Los conceptos que Corder presentó no solo dirigen la atención de la investigación hacia áreas específicas de análisis de errores, sino que también ayudó a los lingüistas a darse cuenta que a pesar de que los errores a veces dificultan la comunicación, también pueden facilitar la adquisición de la segunda lengua. Estos conceptos también han jugado un papel importante en la formación de profesores para ayudarles a identificar, clasificar los errores de los estudiantes y también para ayudarles a construir las técnicas de corrección. Esta última tarea, sin embargo, ha resultado ser bastante compleja y cuestionada hasta el punto de ser considerada como poco efectiva (Truscott, 1996). 


\subsection{2. ¿Qué es el feedback correctivo?}

Primero que todo, el feedback correctivo fue definido, hace ya más de tres décadas, como cualquier reacción del profesor que claramente transforma, se refiere a, o demanda corrección del enunciado del hablante (Chaudron, 1977), veinte años más tarde, Lightbown y Spada (1999) amplían el rango de los actores involucrados en el proceso de feedback, ellas definen el FC como una indicación del profesor, hablante nativo o hablante no-nativo a sus interlocutores de que el uso de la lengua meta es incorrecto. En la actualidad, el FC se encuentra en la literatura especializada bajo diferentes denominaciones que refieren exactamente al mismo concepto: (1) corrective feedback, (2) negative evidence, (3) negative feedback y error treatment (Sheen, 2011). Hoy en día, el FC es considerado como una reacción del profesor que invita al estudiante a poner atención a la precisión gramatical de algo que se ha dicho o escrito (Sheen, 2011); esta definición releva aún más los roles de quienes participan en el proceso de feedback, la función orientadora o indicativa del profesor (tutor) y la de ser capaz de "notar" del estudiante en torno a la precisión gramatical o accuracy.

\subsection{3. ¿Cuál es el propósito de dar feedback acerca de los errores?}

Esta pregunta es muy importante al evaluar los estudios que se han realizado acerca de la corrección de los errores. Estos propósitos pueden variar sustancialmente dependiendo, por ejemplo, de la modalidad del lenguaje; es decir, si el feedback se da en forma oral o en forma escrita. El primero es una estrategia de corrección (Long, 1991, 1996) que apunta a los errores de los estudiantes acerca de su producción en una actividad comunicativa oral (Sheen, 2011), es inmediato y se realiza mediante una comparación en línea (online); en cuanto al segundo, es el que se entrega acerca de los errores que cometen los estudiantes en sus textos escritos (Bitchener \& Ferris, 2012), es diferido y por lo tanto la comparación se realiza fuera de línea (off-line); esto significa que el feedback escrito podría requerir menor carga cognitiva para la memoria que el feedback oral, dado que el feedback entregado en forma escrita va a estar disponible durante un periodo más prolongado 
de tiempo. La mayoría de la investigación en feedback correctivo oral, se ha centrado en la corrección de los errores de forma, con el exclusivo propósito de mejorar la precisión gramatical de elementos lingüísticos focalizados (Iwashita, 2003). Por otra parte, los estudios de feedback correctivo escrito usualmente han tenido una focalización variada, distinta a solamente mejorar la precisión gramatical de los textos escritos de los estudiantes, porque además se han encargado de mejorar la calidad del texto escrito mediante el desarrollo de estrategias de revisión, además del contenido y la organización (Ellis et al. 2008, Ferris, 2010). Esta variabilidad puede explicar, hasta cierto punto, en contraste a la investigación de ASL que ha demostrado que el FC oral es efectivo, porqué la investigación en FC escrito no ha logrado demostrar fehacientemente que el FC escrito conlleva a mejorar la precisión gramatical en la producción de textos escritos nuevos en una L2.

\subsection{4. ¿Cuándo se deben corregir los errores escritos?}

Un tema crucial en la corrección de los errores se refiere a si éstos deben ser corregidos inmediatamente después que ocurren o diferir para más tarde (Sheen, 2011). Hendrickson (1978) se dio cuenta que los profesores de lengua habían rechazado la tesis de evitar los errores a toda costa durante el enfoque audio lingual y aceptaban un amplio rango de ellos y solamente trataban aquellos que resultaban más problemáticos. Hoy en día existe una vez más, mayor consenso en que el FC tiene un rol importante en la precisión gramatical, pero algunos metodólogos sienten que no tiene un lugar relevante en la fluidez, argumentando que los estudiantes no deberían ser interrumpidos cuando hablan (Harmer, 2007). Harmer recomienda corregir los errores en forma diferida hasta que la actividad comunicativa haya sido completada. Este punto es particularmente importante en el FC escrito, ya que en prácticamente todas las ocasiones se realiza en forma diferida, lo que conlleva ciertos beneficios desde el punto de vista del procesamiento de la información, porque el estudiante tiene mucho más tiempo para reflexionar acerca de los errores cometidos y para analizar la distancia que existe con la lengua meta. 


\subsection{5. ¿Cómo se deben corregir los errores escritos?}

Hendrickson (1978) declara la importancia de investigar los métodos de corrección sistemáticos y la entrega de feedback selectivo (focalizado). En relación a esto último, existe diferencia entre la corrección focalizada (limitada a uno o dos categorías de errores) y la no-focalizada (tratamiento de un amplio rango de errores). Aun cuando algunos metodólogos prefieren la corrección no-focalizada, estudios recientes han demostrado que la corrección focalizada es más efectiva (Ellis et al., 2008). Las estrategias que mayoritariamente han sido objeto de discusión e investigación son el FC directo e indirecto (Sheen, 2011). La corrección directa considera la entrega de la forma correcta, mientras que la indirecta es una indicación de que hubo algún error, pero sin entregar la forma correcta. Existe mayor preferencia entre los expertos en producción escrita por el feedback indirecto, argumentando que este tipo de corrección es más efectiva porque requiere que los estudiantes se involucren en actividades de resolución de problemas y de autocorrección porque además demanda mayor procesamiento de la lengua (Ferris, 1999). Sin embargo, los defensores del feedback correctivo directo (Chandler, 2003; Bitchener \& Knoch (2010b) argumentan que solo la corrección directa es capaz de ofrecer a los estudiantes el tipo de información explícita necesaria para probar sus hipótesis acerca de la lengua meta. Estudios recientes (Baker \& Bricker, 2010) han encontrado evidencia empírica que sostiene que los estudiantes corrigieron sus textos en forma más precisa cuando la corrección fue directa y que los estudiantes encontraron dificultades para identificar la corrección indirecta.

\subsection{La actitud de los estudiantes hacia el FC escrito}

La investigación de FC sobre las actitudes de los estudiantes ha sido mayoritariamente descriptiva, catalogando las preferencias y las percepciones de la instrucción gramatical y las distintas maneras de entregar feedback (Sheen, 2011). En un estudio de FC escrito, Leki (1991) examinó las preferencias en la corrección de errores de 100 estudiantes de enseñanza superior; su estudio reveló que la gramática perfecta era uno de los aspectos que los estudiantes mayoritariamente 
valoraban, además de considerar la corrección de los errores como la mejor ayuda que los profesores les podían entregar en este aspecto. Leki también informó que a pesar de la preferencia que tenían los estudiantes por la corrección de los errores, algunos de ellos indicaban que no ponían mayor atención a las correcciones acerca de la precisión gramatical. En cuanto a la preferencia por tipo de FC, $67 \%$ de los estudiantes querían que el profesor les entregara $\mathrm{FC}$ directo ${ }^{2}$ señalando la ubicación del error en conjunto con explicaciones metalingüísticas para ayudarlos a corregir sus errores y ni uno consideró el FC indirecto ${ }^{3}$ como el más apropiado.

En otro estudio, Enginarlar (1993) investigó la opinión de 47 estudiantes acerca de la utilidad y el valor instruccional del FC escrito. El FC se entregó en la corrección de los errores gramaticales, vocabulario y puntuación, utilizando un sistema codificado de corrección. Enginarlar descubrió que los estudiantes no apreciaban los ejercicios de revisión, pero que la mayoría valorizó el FC entregado. Cuando se les preguntó acerca de su preferencia por quién entrega el FC, la mayoría prefirió al profesor.

En la misma línea, en un estudio a gran escala, Schulz (1996) investigó las actitudes de estudiantes universitarios y sus profesores hacia la instrucción gramatical y la corrección de los errores, utilizando un cuestionario que consistía en preguntas de selección múltiple. Ella encontró que la actitud de los estudiantes hacia la instrucción de la gramática y la corrección de los errores era más favorable que las de sus profesores; $90 \%$ de los estudiantes tuvo una actitud positiva hacia la corrección de sus errores, que contrastó con la actitud de sus profesores que no valoraron su propio feedback. En la misma línea de acción, Ancker (2000) investigó la percepción de profesores y estudiantes en 15 países; $25 \%$ de los profesores y $75 \%$ de los estudiantes respondieron positivamente cuando se les preguntó acerca de si los profesores deberían corregir todos los errores cuando usaban inglés. Los profesores estaban preocupados del impacto negativo en la confianza y motivación de los estudiantes, muy en la línea de Krashen (1982) y

2. Cuando se entrega la corrección del error.

3. Cuando se marca el error mediante claves y se les solicita a los estudiantes autocorregirse. 
su constructo del filtro afectivo, mientras que los estudiantes querían ser corregidos porque querían aprender inglés correctamente.

\subsection{La actitud como factor mediador entre la precisión gramatical y el efecto del FC}

A pesar de lo anterior, Truscott (1996) insiste que lo que los estudiantes prefieran o deseen puede no ser realmente lo mejor para la adquisición. Por consiguiente, lo que se necesita son estudios empíricos que además examinen la relación entre las actitudes de los estudiantes hacia la corrección, la gramática y los resultados de la aplicación de FC escrito. Pocos estudios han explorado la relación entre las actitudes de los estudiantes hacia el FC y el aprendizaje resultante de su aplicación. Sheen (2008) estudió el impacto que tienen ciertos factores cognitivos y afectivos en la habilidad de los estudiantes para usar el FC oral y escrito en el proceso de adquisición; los resultados indican que cuatro tipos de FC produjeron resultados de correlación similar en relación con las variables de diferencias individuales, siendo una de ellas la actitud.

Havranek y Cesnik (2001) realizaron un estudio comprensivo ${ }^{4}$ con 207 hablantes alemanes que aprendían inglés como L2. Ellos compararon los efectos del parafraseo, repetición más parafraseo y elicitación, midiendo el éxito de la corrección de errores en el desempeño de los estudiantes en pruebas posteriores. El estudio informó que el feedback correctivo podía beneficiar a los estudiantes que tenían una actitud positiva hacia la corrección de los errores y una alta competencia lingüística.

En resumen, es factible que las actitudes afecten la conducta de los estudiantes, que a la vez influye en el aprendizaje. Sheen (2011) sugiere que se puede hipotetizar que los estudiantes con actitudes positivas hacia el FC y un buen desempeño en la precisión gramatical se benefician más del FC que los que tienen actitudes negativas.

En este sentido, parte del estudio informado en este artículo se centró en la relación existente entre el efecto de tres tipos de FC escrito (no metalingüístico, metalingüístico y mediado por computador) en 
relación a la adquisición de dos estructuras de la lengua inglesa (pasado simple y concordancia sujeto-verbo en presente simple) y las actitudes de los estudiantes hacia el FC escrito; respondiendo a las siguientes preguntas de investigación:

1. ¿Cuál es la actitud que tienen los estudiantes que reciben FC escrito hacia la corrección de errores en el proceso de producción escrita en inglés como L2?

2. ¿Qué relación tiene la actitud de los estudiantes hacia la corrección de los errores con el efecto del FC escrito en la precisión gramatical del uso del pasado simple y la concordancia sujeto-verbo en inglés como L2?

\section{Metodología}

El estudio utilizó un diseño cuasi-experimental de grupos intactos, - G1 feedback correctivo no-metalingüístico $(\mathrm{N}=18), \mathrm{G} 2$ feedback correctivo metalingüístico ( $\mathrm{N}=19), \mathrm{G} 3$ feedback correctivo mediado por computador $(\mathrm{N}=18)$ y $\mathrm{G} 0$ grupo control $(\mathrm{N}=18)$. Los cuatro grupos completaron un pre-test, un postest y un postest diferido. Los tres tests consistieron en un texto escrito en formato de carta y un test de corrección de errores, los cuales fueron aplicados antes de un tratamiento con FC, inmediatamente después y en forma diferida. Al mismo tiempo, los cuatro grupos completaron un cuestionario de actitudes.

En cuanto al tratamiento, los tres grupos experimentales recibieron corrección de los errores en cuatro textos escritos, 2 de los cuales fueron revisiones. Los tres grupos experimentales recibieron corrección directa focalizada ${ }^{5}$ exclusivamente dirigida a errores que involucraban el uso del pasado simple y de concordancia entre sujeto-verbo en la lengua inglesa. Por otra parte, el grupo control sólo recibió un comentario general acerca del contenido de los cuatro textos escritos.

El estudio consideró un análisis de porcentajes de respuesta en relación al cuestionario de actitudes y un análisis de correlación entre 
la variable individual actitud y los puntajes del pretest, postest y postest diferido, con la intención de examinar el rol moderador de la variable sobre los efectos del tratamiento de corrección de errores.

\subsection{Participantes}

95 estudiantes, 46 mujeres y 49 hombres, de tres cursos de $7^{\circ}$ año básico de un colegio particular bilingüe español-inglés de la comuna de San Pedro de la Paz, Concepción, Chile; cuyas edades fluctúan entre 13 y 14 años de edad participaron del estudio.

De la población inicial, 15 de los estudiantes participaron en dos pequeños estudios piloto y 7 estudiantes fueron descartados de la muestra final, debido a que sobrepasaron los 90 puntos, equivalentes al 90\% de precisión gramatical en el pretest ${ }^{6}$. Consecuentemente, la muestra final quedó conformada por 73 sujetos.

\subsection{Instrumentos}

Para cada sesión de evaluación se administró un pretest, postest y postest diferido. Para estos efectos, los estudiantes escribieron una carta. Para que los estudiantes escribieran el texto, se les entregó un estímulo escrito que planteaba una situación relacionada con su entorno académico y a la cual debían reaccionar en forma escrita. Para el pretest, postest y el postest diferido, los estudiantes escribieron un nuevo texto (carta), de la misma dificultad, extensión y duración. El pretest se administró inmediatamente antes de que los estudiantes recibieran la primera tarea de producción escrita. El postest se administró el mismo día que los estudiantes recibieron feedback de su último texto escrito. El postest diferido se administró aproximadamente 5 semanas después.

A los participantes también se les pidió que completaran un cuestionario, basado en Sheen (2006). El propósito de este instrumento fue analizar la actitud de los estudiantes hacia el FC escrito en torno a tres constructos de actitud: (1) preferencias generales acerca del FC

6. Ellis et al. (2008) consideran que un porcentaje superior al $90 \%$ es un indicador de adquisición. 
escrito, (2) reacción hacia la utilidad del FC escrito, y (3) preferencias por tipos de feedback correctivo escrito. Además, las respuestas a este instrumento sirvieron para determinar si existía algún efecto mediador entre la actitud de los estudiantes y el FC escrito. 22 preguntas con escala Likert de 5 puntos (strongly disagree - strongly agree) cubrieron las tres áreas.

\subsection{Métodos de análisis de datos}

Para analizar el tratamiento de FC se obtuvieron puntajes de pretest, postest y postest diferido. Los puntajes fueron calculados mediante 'análisis de ocasiones obligatorias' ${ }^{7}$. En cuanto al cuestionario de actitudes, a la mayoría de las 35 preguntas se les asignaron valores numéricos. Las primeras 13 preguntas entregaron información personal de los estudiantes. Al resto de los ítems se les aplicó una escala Likert de 5 puntos comenzando con strongly disagree (totalmente en desacuerdo), uno (1); hasta strongly agree (totalmente de acuerdo), cinco (5). La información porcentual se calculó mediante una tabla de frecuencias de respuestas de cada ítem del cuestionario. Se realizó la estadística descriptiva de los puntajes de las actitudes de los estudiantes hacia la corrección de los errores y se analizó cada set de puntajes para investigar diferencias grupales utilizando un análisis de varianza, ANOVA de un factor. Adicionalmente se calcularon coeficientes de correlación de Pearson entre los puntajes del cuestionario de actitudes y las ganancias de los puntajes de los tests de producción escrita a corto y largo plazo. También se utilizó un análisis de covarianza ANCOVA de medidas repetidas para analizar el efecto mediador de la actitud en relación a los efectos de los tipos de FC escrito del estudio.

\section{Análisis de resultados}

Antes de presentar los resultados para las dos preguntas de investigación, en la tabla 1 se muestra la estadística descriptiva para

7. Todas las ocasiones obligatorias de uso del pasado simple y de la concordancia sujetoverbo fueron identificadas. Cada ocasión fue examinada para ver la correcta aplicación ambas estructuras (Ellis \& Barkhuizen, 2005). 
el total de los puntajes del pretest, postest y postest diferido, con la correspondiente desviación estándar.

Tabla 1 - Medias grupales y desviación estándar de los puntajes de los tests

\begin{tabular}{|c|c|c|c|c|c|c|c|c|}
\hline Grupo & Pretest & $\mathrm{DE}$ & Postest & & $\mathrm{DE}$ & Postest diferido & & $\mathrm{DE}$ \\
\hline G0 & 61,0 & 9,9 & 61,6 & b & 15,1 & 56,9 & b & 12,2 \\
\hline G1 & 63,0 & 10,9 & 82,7 & a & 10,8 & 71,2 & a & 13,2 \\
\hline G2 & 63,0 & 13,7 & 81,0 & a & 10,9 & 81,4 & a & 7,3 \\
\hline G3 & 60,9 & 12,6 & 78,7 & a & 13,3 & 79,8 & a & 10,9 \\
\hline Valor $\mathrm{p}$ & 914 & &, 000 & & &, 000 & & \\
\hline
\end{tabular}

Esta tabla muestra que todos los grupos ${ }^{8}$ obtuvieron un puntaje superior a 60 puntos en el pretest; mediante un análisis de varianza de un factor se pudo establecer que no existían diferencias estadísticamente significativas entre los grupos del estudio, $F(3,69)=, 174, \mathrm{p}=, 914$. Además se observa que solo los tres grupos experimentales fueron capaces de incrementar la precisión gramatical entre el pretest y el postest estableciendo diferencias estadísticamente significativas con el grupo control, $F(3,69)=10,729, \mathrm{p}<, 001$; así como entre pretest y postest diferido, $F(3,69)=18,779, \mathrm{p}<, 001$. Sin embargo, solo los participantes de los grupos G2 y G3 fueron capaces de incrementar la precisión gramatical después del pretest, y sostener esta ganancia en precisión en el postest diferido.

La figura 3 muestra una representación visual del promedio de los puntajes para cada una de las instancias de evaluación - pretest (T0), postest inmediato (T1) y postest diferido (T2) - para cada uno de los grupos del estudio.

8. G1 feedback correctivo no-metalingüístico $(\mathrm{N}=18), \mathrm{G} 2$ feedback correctivo metalingüístico $(\mathrm{N}=19), \mathrm{G} 3$ feedback correctivo mediado por computador $(\mathrm{N}=18)$ y $\mathrm{G} 0$ grupo control ( $\mathrm{N}=18)$ 


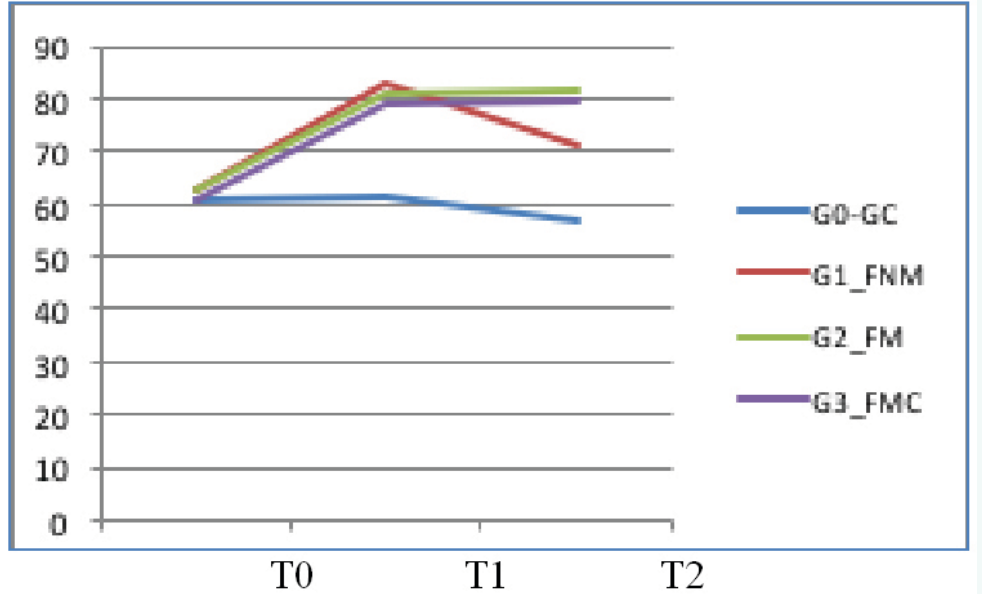

Figura 3 - Puntajes de los tests escritos de cada uno de los grupos del estudio

\subsection{Análisis de resultados del cuestionario de actitudes hacia el FC escrito}

La pregunta 1 consistió en averiguar cuál era la actitud de los estudiantes que reciben $\mathrm{FC}$ escrito hacia la corrección de errores gramaticales en inglés como L2. En términos generales, los resultados del cuestionario, corroboran lo informado por otros estudios (Schulz, 1996; Sheen, 2006, 2008) demostrando que los estudiantes aprecian y esperan recibir FC de sus trabajos escritos, refuerzan lo argumentado por Ferris (1999) y se contraponen a lo expuesto por Truscott (1996) en relación a la importancia que tienen las preferencias de los estudiantes hacia la corrección de los errores. Al consultarles directamente acerca de aquello, un $73 \%$ de los estudiantes del estudio está de acuerdo en recibir FC escrito o corrección escrita. Los estudiantes también parecen entender la importancia de la precisión gramatical (75\%), en contraste al $6 \%$ que no lo está; esta percepción resulta interesante porque a pesar de que el contexto de aprendizaje se caracteriza por estar centrado en el contenido, la organización y el desarrollo de habilidades escritas, la mayoría de los estudiantes ha reconocido que el uso de la lengua también tiene un papel importante en el proceso de escritura. 
Al consultarles acerca de las preferencias generales de FC, la mayoría de los estudiantes prefieren ser corregidos por su profesor (69\%), lo que ha resultado muy similar a lo informado por Enginarlar (1993) y no consideran sentirse frustrados cuando su profesor los corrige (58\%). La responsabilidad en la corrección de los errores ha sido un tema de discusión desde hace más de 20 años (Sheen, 2011; Bitchener \& Ferris, 2012); por lo tanto, la evidencia que arrojan las preferencias de los estudiantes en función de este tema releva el rol del profesor en esta tarea. Los estudiantes también demuestran un grado de satisfacción al ser corregidos $(70 \%)$ y no son indiferentes a cometer errores en sus trabajos escritos, ya que sienten por una parte la necesidad de saber en qué se equivocan (81\%), así como también reflexionar acerca de los errores (74\%) antes de escribir un nuevo texto. En su argumento contra el FC escrito, Truscott (1996) argumenta que éste es dañino; esto se fundamenta en que la corrección de los errores afecta al filtro afectivo (Krashen, 1982) ya que los estudiantes que reciben correcciones por parte de sus profesores perderían confianza y seguridad en ellos mismos y de esta manera el proceso de adquisición se vería afectado. De acuerdo a lo informado por los propios estudiantes entonces, es evidente que muy por el contrario a lo expuesto por Truscott, el ser corregidos les entrega seguridad y les permite además reflexionar acerca de los errores que comenten, lo que resulta muy importante para futuras tareas escritas. Lo que sí afecta negativamente a los estudiantes es repetir el mismo error (58\%); en ese sentido entonces, basado en el mismo argumento que ha planteado Truscott (1996), el FC más que elevar el nivel del filtro afectivo puede tener la capacidad de rebajarlo, y por consiguiente ayudar a los estudiantes a superar la frustración de equivocarse.

En cuanto a la reacción hacia el FC y su utilidad, la mayoría de los estudiantes (84\%) admite que las correcciones les ayudaron a mejorar su producción escrita; del mismo modo, un $72 \%$ cree que el FC ayuda a mejorar la calidad de los textos; los estudiantes no solamente aprecian ser corregidos por sus profesores, sino que también encuentran un valor práctico y útil en la corrección de los errores en cuanto al proceso de escritura. Si bien es cierto los hablantes no necesariamente son los mejores jueces para saber qué es lo que más necesitan (Truscott, 1996), ellos sugieren que esta práctica puede influir en su éxito o fracaso académico. Ferris (2004) sostiene que la investigación acerca 
de cómo los estudiantes visualizan el FC predice que la presencia de la corrección de los errores puede llegar a ser positiva y que su ausencia puede llegar a ser dañina. A pesar de lo anterior, llama la atención el porcentaje de sujetos (55\%) que no estima que la corrección sea capaz de evitar la repetición del mismo error en la escritura de textos nuevos. Es probable que los estudiantes se den cuenta que a pesar de su deseo de ser corregidos y encontrar que la corrección es positiva para su trabajo académico, existen ciertas estructuras erróneas en su escritura que definitivamente no logran superar. De acuerdo a lo argumentado en este estudio, es probable que esta última situación se deba a la incapacidad de asimilar las correcciones debido a las falencias que existen en la manera en que dicha corrección se ha llevado a cabo históricamente y que ha sido tema ampliamente analizado (Lillo, 2014); a saber, poca sistematicidad en los métodos de corrección que muchas veces se basan en la intuición y no en la teoría, la investigación y la práctica sistemática. El cuestionario también muestra que un 59\% de los estudiantes reconoce poner atención a las correcciones de sus tareas; y el 70\% estima que el poner atención a este feedback les ayuda a mejorar su gramática. En este mismo aspecto, al consultar acerca del hábito de la corrección después de recibir el feedback, el 60\% de los sujetos respondió que sí lo hace y un 14\% que no; estos porcentajes de aprobación son bastante importantes, pero esperables en un contexto de escritura como proceso, porque es muy probable que el hecho de tener que revisar y editar el texto demande que necesariamente los estudiantes tengan que revisar las correcciones para reescribirlo.

Con respecto a las preferencias específicas por tipos de FC; lo primero que el cuestionario indagó fue acerca de la dicotomía que existe en la cantidad de información que entrega el FC, lo que en este estudio se ha definido como FC directo e indirecto; en ese sentido, el 77\% de los estudiantes expresó preferencia por el feedback directo con explicación por parte del profesor, el $66 \%$ desaprueba que su profesor les entregue feedback directo sin una explicación acerca del error cometido y un $71 \%$ considera importante que el feedback entregado también se haga en forma personalizada; estos resultados son muy similares a los encontrados por Leki (1991), indicando que los estudiantes prefieren un FC directo que entregue información metalingüística por sobre el que no la entrega. En cuanto al feedback indirecto, solo un $46 \%$ de los estudiantes lo desea. La literatura especializada en FC escrito (Chandler, 
2003 ) indica que los profesores e investigadores han favorecido el uso de feedback correctivo indirecto con un marcado énfasis en el proceso de revisión y edición; en contraste, pocos estudios han investigado el feedback directo, comparando un grupo experimental con un grupo control que no recibe feedback, como se ha realizado en esta investigación. Por consiguiente, esta preferencia por un tipo de feedback directo, resulta importante como contribución a la discusión de la efectividad de estos dos tipos de FC. Se ha discutido que aquel feedback que es menos directo produce una mayor profundidad en el procesamiento cognitivo de los errores cometidos en el proceso de adquisición en la L2, pero también se ha argumentado que muchas veces los estudiantes no son capaces de comprender las claves que les entregan sus profesores para la autocorrección de los errores y en ese sentido un feedback más directo va a ser definitivamente más efectivo. La segunda dicotomía que aborda el cuestionario, es la que tiene relación con el número de errores a corregir en un tratamiento, lo que se conoce técnicamente como feedback focalizado y no focalizado. En relación con la focalización del feedback, un 70\% de los estudiantes prefiere que su profesor les corrija todos los errores cometidos y solo un $53 \%$ que la corrección se concentre en una o dos categorías; es probable que esta preferencia esté asociada a lo que habitualmente experimentan los estudiantes debido a que los profesores de segundas lenguas tienden a corregir el máximo de los errores de sus estudiantes de manera poco sistemática (Truscott, 1996), sin criterios claros y de manera intuitiva. De todas maneras, es importante tener en consideración que los resultados en torno a este punto también contribuyen a la discusión en cuanto a la focalización de la corrección de los errores (Bitchener 2008; Bitchener \& Ferris; 2012), ya que la investigación robusta en adquisición de segundas lenguas estima que el tratamiento no debiera superar dos o tres categorías gramaticales, mientras que la investigación en composición escrita considera que ese número es poco práctico en un contexto de sala de clases. Ellis et al. (2008) sugieren un equilibrio entre ambas posturas, lo que además va a depender de factores propios de dificultad relacionados con las estructuras seleccionadas porque no todas tienen la misma condición de reparación.

En relación a la corrección realizada con tecnología, un $60 \%$ de los estudiantes aprueba que se utilice en este proceso, lo que es esperable, tal vez por la relevancia que tiene para los nativos digitales la tecnología 
en su vida cotidiana, aunque no es algo que suceda habitualmente como recurso asociado a la producción escrita en la L2; por lo demás, solamente fue utilizada como estrategia de tratamiento en un solo grupo de investigación y esto de alguna manera condiciona la preferencia por esta modalidad de feedback.

\subsection{Correlación entre puntaje del test de actitud y ganancias de puntajes de los tests a corto y largo plazo.}

La segunda pregunta de investigación consistió en averiguar la relación que tiene la actitud con el efecto del FC. A continuación se presenta la estadística descriptiva para los puntajes de las actitudes de los estudiantes hacia la corrección de errores y la correlación entre la ganancia en los puntajes a corto y largo plazo en los tests de producción escrita (entre pretest y postest inmediato, entre pretest y postest diferido)

La tabla 2 muestra la estadística descriptiva de los puntajes de actitudes de los estudiantes hacia la corrección de los errores escritos. El puntaje promedio para los cuatro grupos fluctúa entre 3,33 y 4,01; el G0 alcanza el menor puntaje y el G3 alcanza el mayor. Al mismo tiempo, una ANOVA de un factor, $F(3,69)=12,297, \mathrm{p}<, 001$ indica que la diferencia entre los grupos experimentales y el grupo control es estadísticamente significativa.

Tabla 2 - Estadística descriptiva para los puntajes del cuestionario de actitudes

\begin{tabular}{cccccc}
\hline & N & Media & DT & Mínimo & Máximo \\
\hline G0 & 18 & 3,3278 &, 48211 & 2,43 & 4,24 \\
G1 & 18 & 3,7383 &, 46495 & 2,67 & 4,52 \\
G2 & 19 & 3,9726 &, 28221 & 3,29 & 4,52 \\
G3 & 18 & 4,0133 &, 24350 & 3,65 & 4,33 \\
Total & 73 & 3,7659 &, 46324 & 2,43 & 4,52 \\
\hline
\end{tabular}

La tabla 3 muestra cuál es la asociación que existe entre los puntajes generales del cuestionario de actitudes y las ganancias a corto y largo plazo para cada uno de los grupos del estudio en relación al conjunto 
de estructuras. El análisis de correlación de Pearson revela que hubo asociación positiva para G3 a corto plazo, $r=, 613 ; p=, 007$. Pero solamente para el grupo G2, a corto plazo, $r=487 ; p=, 034$; así como también a largo plazo, $\mathrm{r}=, 639 ; \mathrm{p}<, 001$.

Tabla 3 - Correlación entre la ganancia en puntajes de los tests de producción escrita y los puntajes del cuestionario de actitudes por grupo

\begin{tabular}{ccc}
\hline & GANACIAS A CORTO PLAZO & GANACIAS A LARGO PLAZO \\
\hline G0 &, 071 &, 268 \\
&, 780 &, 282 \\
G1 &, 418 &, 282 \\
&, 084 &, 256 \\
G2 &, $487^{*}$ &, $639^{* *}$ \\
&, 034 &, 000 \\
G3 &, $613^{* *}$ &, 389 \\
&, 007 &, 111 \\
\hline
\end{tabular}

Para observar hasta qué punto las actitudes de los estudiantes influyen en el efecto del FC escrito, se presentan los resultados de un análisis de medidas repetidas ANCOVA (Análisis de covarianza), con la actitud general hacia la corrección de errores como covariable. La tabla 5 muestra los resultados de este análisis.

Como se puede observar en la tabla 4 , hubo un efecto significativo para las actitudes entre los grupos; $F(1,68)=4,391, \mathrm{p}=, 040$ indicando que la actitud de los grupos experimentales difiere significativamente de la del grupo control. La tabla también arroja evidencia estadísticamente significativa para el tratamiento de FC, $F(3,68)=4,221, \mathrm{p}=, 008$. La tabla no entrega evidencia estadísticamente significativa del efecto Tiempo, pero sí de la interacción Tiempo * Tratamiento, $F(6,136)$ $=2,250, \mathrm{p}=, 042$ y también para la interacción Tiempo*Actitudes $F$ $(2,136)=3,481, \mathrm{p}=, 034$. 
Tabla 4 - ANCOVA de medidas repetidas para los puntajes de los tests y actitudes

\begin{tabular}{lccc}
\hline Origen & $\mathrm{gl}$ & $\mathrm{F}$ & Sig. \\
\hline Inter estudiantes & & & \\
Actitudes & 1 & 4,391 &, 040 \\
FC & 3 & 4,221 &, 008 \\
Error & 68 & & \\
Entre estudiantes & & & \\
Tiempo & 2 &, 923 &, 400 \\
Tiempo * Actitudes & 2 & 3,481 &, 034 \\
Tiempo * FC & 6 & 2,250 &, 042 \\
Error(Tiempo) & 136 & & \\
\hline
\end{tabular}

Como se ha observado en esta sección, los resultados de la estadística descriptiva del cuestionario de actitudes indican que existe una diferencia en los puntajes del test de actitudes entre los grupos del estudio. El análisis de ANOVA demostró que esta diferencia es estadísticamente significativa, lo que indica que los grupos experimentales superaron al grupo control en los puntajes de las respuestas del cuestionario. Respecto a las correlaciones entre los puntajes de los tests y los puntajes del cuestionario de actitudes, los resultados indicaron que hubo una correlación positiva entre la actitud hacia el FC escrito y las ganancias de los puntajes de los test de producción escrita a corto plazo (entre pretest y postest) y a largo plazo (entre pretest y postest diferido). Sin embargo, al examinar la correlación por grupos, es posible darse cuenta que no hubo relación entre las ganancias de los puntajes y la actitud hacia la corrección de los errores en el G0. Se puede esperar una relación entre ambos solamente si los estudiantes están conscientes de que están siendo corregidos y qué es lo que está siendo corregido (Sheen, 2011), obviamente los estudiantes del grupo control no fueron conscientes de aquello. Esta condición sí estuvo presente en el resto de los grupos y en estos casos se encontraron correlaciones entre las actitudes de los participantes y las ganancias de los puntajes de los tests con significancia estadística. La actitud hacia el FC escrito fue mucho más fuerte en el caso de los grupos que recibieron mayor información metalingüística, hubo correlaciones estadísticamente significativas a corto y largo para el 
G2, corroborando los resultados de Sheen (2006) que indican que las actitudes tienen un efecto mediador sobre el FC escrito. Sin embargo, mientras las actitudes tuvieron un rol importante en los grupos G2 y G3 en menor medida, no sucedió lo mismo con el G1. Es interesante ver que el hecho de recibir una corrección directa sin información adicional no logra el mismo efecto de manera consistente; la explicación una vez más se puede sustentar en que no se puede esperar que la actitud tenga un efecto mediador si los estudiantes no están conscientes de que están siendo corregidos. Esto puede tener implicaciones pedagógicas importantes en torno a las consideraciones que tienen que tener los profesores al corregir. En resumen, los resultados demuestran que el FC es beneficioso para los estudiantes que tienen una actitud positiva hacia la corrección de los errores en un mayor grado que aquellos que no la tienen. Esto ha sido corroborado por el análisis de covarianza ANCOVA que indicó que hubo un efecto significativo para las actitudes entre los grupos, demostrando que la actitud de los grupos experimentales, específicamente aquellos que recibieron mayor información explícita, difiere significativamente a la del grupo control en el tiempo.

Los estudiantes valoran el feedback principalmente por el rol potencial que tiene en el desarrollo de la lengua. Por ende, la consideración de la actitud de los estudiantes es crucial si el feedback correctivo escrito va a ser aprovechado como estrategia de aprendizaje de la L2.

\section{Conclusiones}

Truscott (1996) no solamente ha cuestionado la efectividad del FC escrito, sino que además ha minimizado la importancia de la necesidad que expresan los estudiantes por la corrección de sus errores. Gass y Selinker (2008) han informado que las actitudes de los estudiantes hacia la corrección de los errores, entre otros factores, pueden afectar los resultados de aprendizaje. Algunos estudios han examinado las preferencias de los estudiantes por la corrección de sus errores escritos, los estudios de Havranek y Cesnik (2001) y Sheen $(2006,2008)$ no solamente han demostrado que los estudiantes desean ser corregidos, sino que además han entregado evidencia importante de que la actitud hacia la corrección de los errores puede tener un impacto en la habilidad 
de los estudiantes para usar el FC escrito en el proceso de adquisición y aprendizaje de una segunda lengua. En esta misma línea, el presente estudio informa que el feedback correctivo, especialmente el que genera consciencia metalingüística, puede beneficiar más a los estudiantes que tienen una actitud positiva hacia la corrección de los errores que aquellos que no la tienen.

Uno de los argumentos de Truscott en contra del FC se basa también fuertemente en el daño que éste puede causar a los hablantes de una L2; esta postura se fundamenta en la hipótesis del filtro afectivo (Krashen, 1982) que establece la necesidad de realizar el proceso de adquisición cuando se consigue un sentimiento de confianza y seguridad en sí mismo para comunicarse, lo que sería imposible que ocurriera cuando se corrigen los errores dada la ansiedad que se genera. Sin embargo, los resultados de este estudio revelan que los estudiantes desean ser corregidos en la producción de sus textos escritos, al mismo tiempo que mejoraron la precisión gramatical de las estructuras tratadas con FC. Truscott (1996) no encuentra que la actitud positiva de los estudiantes sea importante a la hora de tomar decisiones en la entrega de FC; sin embargo, contrario a esto, en este estudio se entrega evidencia de que la actitud de los estudiantes hacia el FC tiene cierta asociación con los efectos de usar ciertos tipos de feedback. Es importante que los estudios futuros consideren esta variable individual, así como también otras que generalmente están ligadas al aprendizaje de una L2 como lo son la motivación y la aptitud.

Recebido em: 24/03/2016

Aprovado em: 22/08/2017

E-mail: jlillo@ucsc.cl

\section{Referencias bibliográficas}

ANCKER, William. 2000. Errors and corrective feedback: Updated theory and classroom practice. English Teaching Forum, 8 (4), p. 20-25.

BAKER, Wendy \& RACHEL, Bricker. 2010. The effects of direct and indirect speech acts on native English and ESL speakers' perception of teacher written feedback. System 38, p. 75-84.

BITCHENER, John. 2008. Evidence in support of written corrective feedback. Journal of Second Language Writing, 17 (2), p. 102-118. 
Bitchener, John \& Ute, Knoch. 2010b. The contribution of written corrective feedback to language development: A ten-month investigation. Applied Linguistics, 31, (2), p. 193-214.

Bitchener, John \& Dana Ferris. 2012. Written corrective feedback in second language acquisition. New York: Routledge.

CArroll, John. 1961. Fundamental considerations in testing for English language proficiency of foreign students. In: Allen, Harold (ed.). Teaching English as a second language. New York: McGraw Hill. p. 364-372.

ChANDLER, Jean. 2003. The efficacy of various kinds of error feedback for improvement in the accuracy and fluency of L2 student writing. Journal of Second Language Writing, 12 (3), p. 267-296.

Chaudron, Craig. 1977. A descriptive model of discourse in the corrective treatment of learners' errors. Language learning, 27, (1), p. 29-46.

CORDER, Stephen. 1967. The significance of learners' errors. International Review of Applied Linguistics, 5, p. 161-170.

Ellis, Rod. 1994. The study of second language acquisition. Oxford: Oxford University Press.

Ellis, Rod \& GARY, Barkhuizen. 2005. Analyzing learner language. Oxford: Oxford University Press.

Eluis, Rod; Younghee SheEn, Mihoko Murakami \& Takashima Hide. 2008. The effects of focused and unfocused written corrective feedback in English as a foreign language context. System, 36, p. 353 - 371.

ENGINARLAR, Hüsnü. 1993. Student response to teacher written feedback in EFL writing. System, 21, p. 193-204.

FERRIS, Dana. 1999. The case for grammar correction in L2 writing classes: A response to Truscott 1996. Journal of Second Language Writing, 8, p. $1-10$.

FERrIS, Dana. 2004. The "grammar correction" debate in 12 writing: Where are we, and where do we go from here? (and what do we do in the meantime ... .?). Journal of Second Language Writing, 13, p. 49-62.

FERris, Dana. 2010. Second language writing research and written corrective feedback. Studies in Second Language Acquisition, 32, p. 181-201.

Gass, Susan \& Larry Selinker. 2008. Second language acquisition: An introductory course. New York, NY: Routledge.

HARmer, Jeremy. 2007. The practice of English language teaching. London: Longman.

Havranek, Gertraud \& Hermann Cesnik. 2001. Factors affecting the success of corrective feedback. In: Foster-Cohen, Susan \& Anna Nizegorodzew (eds.). EUROSLA Yearbook, 1. Philadelphia: John Benjamins Publishing Company, p. 99-122. 
HENDRICKSON, James. 1978. Error correction in foreign language teaching: Recent research and practice. The Modern Language Journal. 62, p. 387-398.

IWASHITA, Noriko. 2003. Negative feedback and positive evidence in taskbased interaction: Differential effects of L2 development. Studies in Second Language Acquisition, 25, p. 1-36.

Krashen, Stephen. 1982. Principles and practices in second language acquisition. Oxford: Pergamon Press.

LEKI, Ilona. 1991. The preferences of ESL students for error correction in college-level writing classes. Foreign Language Annals, 24 (3), p. 203-218.

Lillo, Jorge. 2014. Efecto del feedback correctivo escrito directo focalizado, en el proceso de producción de textos escritos en inglés como L2. Tesis de doctorado no publicada. Universidad de Concepción, Chile.

Lightbown, Patsy \& Nina, Spada. 1990. Focus-on-form and corrective feedback in communicative language teaching: Effects on second language acquisition. Studies in Second Language Acquisition, 12, p. 429-448.

Long, Michel. 1991. Focus on form: A design feature in language teaching methodology. En K. De Bot, R. Ginsberg \& C. Kramsch (Eds.). Foreign language research in cross-cultural perspective (pp. 39-52). Philadelphia: John Benjamins Publishing Company.

LonG, Michel. 1996. The role of the linguistic environment in second language acquisition. Handbook of second language acquisition, 26, p. 413-468.

SELINKER, Larry. 1972 Interlanguage. International Review of Applied Linguistics, 10, p. 209-231.

Schulz, Renate. 1996. Focus on form in the foreign language classroom: Students and teachers views on error correction and the role of grammar. Foreign Language Annals, 29, p. 343-364.

SHEEN, Younghee. 2006. Corrective feedback, individual differences, and the acquisition of English articles by second language learners. Tesis de doctorado no publicada. University of Nottingham, United Kingdom.

SHEEN, Younghee. 2008. Recast, language anxiety, modified output and L2 learning. Language learning, 58, p. 835-874.

SHEEN, Younghee. 2011. Corrective Feedback Individual Differences and Second Language Learning. New York: Springster.

TRUSCOTT, John. 1996. Review article. The case against grammar correction in 12 writing classes. Language Learning, 46, p. 327-369. 